\title{
Médiévales
}

Langues, Textes, Histoire

67 | automne 2014

Histoires de Bohême

\section{Mariages clandestins dans la Suède médiévale. Le témoignage des statuts synodaux}

Clandestine Marriages in the Synodal Statutes of Medieval Sweden

\section{Charlotte Christensen-Nugues}

\section{(2) OpenEdition}

1 Journals

\section{Édition électronique}

URL : https://journals.openedition.org/medievales/7449

DOI : 10.4000/medievales.7449

ISSN : $1777-5892$

Éditeur

Presses universitaires de Vincennes

\section{Édition imprimée}

Date de publication : 31 décembre 2014

Pagination : 169-186

ISBN : 978-2-84292-422-5

ISSN : 0751-2708

\section{Référence électronique}

Charlotte Christensen-Nugues, « Mariages clandestins dans la Suède médiévale. Le témoignage des statuts synodaux », Médiévales [En ligne], 67 | automne 2014, mis en ligne le 31 décembre 2016, consulté le 22 avril 2022. URL : http://journals.openedition.org/medievales/7449 ; DOI : https:// doi.org/10.4000/medievales.7449 
Charlotte Christensen-Nugues

\section{Mariages clandestins dans la Suède médiévale Le témoignage des statuts synodaux}

Le mariage se contracte par le seul consentement et n'est pas invalide parce qu'il ne respecte pas les coutumes du pays.

Décrétales de Grégoire IX, IV. 1. $1^{1}$.

Si un homme et une femme veulent se marier à l'Église, ils doivent le faire savoir au prêtre de leur paroisse. Le prêtre doit publier trois dimanches à la porte de l'église que le mari et la femme sont fiancés conformément au droit de la province et qu'ils veulent faire bénir leur union conformément au droit de l'Église.

Loi d'Upland, Livre de l'Église, § $\mathrm{XV}^{2}$.

À partir de la deuxième moitié du xII ${ }^{\mathrm{e}}$ siècle, le droit canonique médiéval établit le consentement mutuel comme le seul acte nécessaire pour créer un mariage valide. Une cérémonie publique, la présence de témoins et la bénédiction d'un prêtre étaient souhaitables pour sa «dignité et solennité ${ }^{3}$ », mais n'étaient pas nécessaires à sa validité. On pouvait donc parfaitement contracter un mariage secrètement. Ces mariages, dits clandestins, représentaient cependant un problème majeur pour la législation matrimoniale de l'Église médiévale: d'une part, leur réalité était difficile à prouver si l'une des parties contestait le mariage, d'autre part, sans publicité préalable, d'éventuels empêchements étaient malaisés à

1. «Matrimonium solo consensu contrahentur, nec invalidatur, si consuetudo patriae non servetur» (Decretales Gregorii IX, éd. E. FrIEDBERG, Corpus Iuris Canonici, t. II, Leipzig, 1881).

2. «Nu wil man ok konæ samæn wighiæs. per aghu soknæ prästi sinum til at sighiæ præster a pæt vm pre sunnudaghæ liusæ. i. kirkiu dorum. at pe hion fast æru mz lanzlaghum. ok per wiliæ mz kirkiu ræt samæn wighiæs » (Upplandslagen enligt Cod. Holm. B 199 och 1607 års utgåva, éd.S. Henning, Uppsala, 1967, p. 42).

3. Pierre Lombard, Sententiae, 4, Dist. 28, c. 2 : « ad decorem et solemnitatem» (PL 192, col. 815). 
découvrir. Les rites ecclésiastiques du mariage se sont développés lentement et ce n'est qu' au XII siècle que commence à se mettre en place une véritable liturgie du mariage ${ }^{4}$. Cette dernière n'est pas obligatoire pour autant. Aucun acte autre que le consentement des époux n'est nécessaire; la présence de témoins ou la bénédiction d'un prêtre peut avoir de l'importance pour la légalité de l'union, mais pas pour sa validité. Un mariage peut, en réalité, se dérouler de façons très différentes: en étant plus ou moins public et en mêlant les rites profanes et religieux. Comme Charles M. de la Roncière l'a bien fait remarquer, la doctrine ecclésiastique du consensus, en insistant sur la simple volonté des contractants, a rendu possible un «florilège des scénarios inattendus improvisés par des jeunes gens pour se rencontrer puis pour s'épouser, en totale discordance avec les cheminements officiels sagement élaborés par les géniteurs aristocratiques et bourgeois et sacralisés par le clergé ${ }^{5} »$. Cette situation a persisté tout au long du Moyen Âge et ce n'est qu'auconcile de Trente (1563) que l'Église a imposé des règles strictes de solennité et de publicité 6 .

L'absence de règles strictes a favorisé de grandes divergences entre les différentes parties de l'Europe. Par exemple, on n'a guère pu imposer la présence du clergé dans le nord de l'Italie, puisque les mariages se faisaient habituellement devant le notaire ${ }^{7}$. Dans des régions tardivement christianisées, telles que la Scandinavie, les pratiques anciennes ont continué à influencer la formation du mariage. Les traditions et les règles séculières ont subsisté bien après la christianisation et se sont ensuite mêlées à la législation ecclésiastique. Ce phénomène apparaît clairement dans la législation diocésaine de la Suède médiévale, et notamment au sujet des mariages clandestins

En Europe occidentale, les mariages clandestins ont constitué une préoccupation constante des diocèses. Les statuts synodaux français de cette époque en traitent abondamment, et il en est de même dans la plupart des autres pays européens. Dans ce contexte, les statuts suédois se distinguent par la place relativement modeste accordée à ce problème. En comparaison avec d'autres pays, ces statuts mentionnent peu la clandestinité et, quand ils le font, ils mettent surtout en cause l'absence de cérémonie religieuse. Dans

4. Pour le développement de la liturgie matrimoniale en France, voir J.-B.Molin, P. Mutembe, Le Rituel du mariage en France du XII au XVI' siècle, Paris, 1974.

5. C. M. DE LA RONCIÈRE, «Rites et idéaux chrétiens face aux pratiques séculaires», dans S. Melchior-Bonnet et C. Salles éd., Histoire du mariage, Paris, 2009, p. 380.

6. Pour les débats sur la clandestinité au concile de Trente, voir C.CHRISTENSEN-Nugues, «Parental Authority and Freedom of Choice: The Debate on Clandestinity and Parental Consent at the Council of Trent (1545-1563)», The Sixteenth Century Journal, 45/1 (2014), p. 45-72.

7. C. KLAPISCH-ZuBER, «Zacharie, ou le père évincé. Les rites nuptiaux toscans entre Giotto et le concile de Trente», Annales, 34 (1979), p. 1216-1243. la suite de cet article, nous analysons ces différences et nous explorerons la tradition légale suédoise pour en déterminer les raisons possibles.

Le mariage et la législation matrimoniale dans la Suède médiévale ont suscité un intérêt croissant ces dernières décennies ${ }^{8}$. Le témoignage des statuts synodaux dans ce contexte, et les différences entre les statuts suédois et ceux d'autres pays européens n'ont cependant été que peu étudiées. Lizzie Carlsson insiste sur le fait que l'introduction du droit matrimonial ecclésiastique fut particulièrement difficile en Suède. Cependant, elle ne relève pas de différences entre les statuts synodaux suédois et ceux d'autres pays européens au sujet des mariages clandestins ${ }^{9}$. Mia Korpiola, en revanche, note que les statuts suédois insistent moins sur les mariages clandestins que ceux de beaucoup d'autres pays. Elle attribue ceci au fait que le droit séculier autorisait des mariages sans l'intervention de l'Église et que l'Église suédoise n'avait pas vraiment les moyens de s'opposer au droit sur ce point ${ }^{10}$. Mia Korpiola ne fait pas, à ce sujet, la distinction entre mariages clandestins, dans le sens de mariages secrets, et ceux sans cérémonie religieuse. Cette distinction est pourtant importante dans une perspective comparatiste : c'est avant tout par la quasi-absence de références aux mariages secrets que les statuts suédois sortent de la norme.

À quelques exceptions près, les statuts synodaux n'apportent aucun point de vue original ni grandes réflexions théoriques sur le droit canonique. En revanche, comme Gabriel Le Bras le fait remarquer, ils ont le mérite de «nous faire connaître l'adaptation du droit commun aux populations diverses de la Chrétienté, de nous laisser entrevoir les réalités de la vie quotidienne ${ }^{11} »$. Les statuts synodaux sont des adaptations locales des règles du droit canonique, règles qui naturellement ne pouvaient pas tenir compte de la multitude de spécificités des différentes parties de la Chrétienté. Certaines questions, abondamment débattues par les canonistes et théologiens, ne sont guère mentionnées dans les statuts, tandis que d'autres ont d'abord été soulevées au niveau provincial et diocésain, avant

8. Voir, entre autres, L.CARLSSON, « Jag giver dig min dotter». Trolovning och äktenskap $i$ den svenska kvinnans äldre historia, 2 vol., Lund, 1965 \& 1972; B. SAWYER, Kvinnor och familj i det forn- och medeltida Skandinavien, Skara, 1992; C. Christensen-Nugues, «Äktenskap och familj», dans J.Christensson éd., Signums svenska kulturhistoria, vol.1, Medeltiden, Lund, 2004, p. 295-333; M.Korpiola, Between Betrothal and Bedding: The

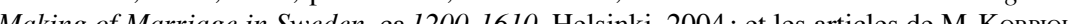
Making of Mariage in Sweden, ca 1200-1610, Helsink, 2004; et les aticles de M. KorPIOL 2000

9. L. CARLSSON, «Jag giver dig min dotter »..., vol. 2, p. 103-105.

10. M. Korpiola, « Matrimonial Law in Late Medieval Swedish Synodal and Provincial Statutes», communication présentée à IMC Leeds, 2001.

11. G. Le Bras, «Préface», dans A. Artonne, L. Guizard et O.Pontal, éd., Répertoire des statuts synodaux des diocèses de l'ancienne France $d u$ XIII à la fin $d u$ XVIII siècle, Paris, 1963 , p. 7 . 
de l'être par le droit commun. C'est le cas des mariages clandestins, où la publication de bans et la présence du prêtre ont d'abord été prescrites au niveau local, par exemple dans les statuts de Westminster (1076 et 1175) ${ }^{12}$, les statuts de Lenzig (1197) ${ }^{13}$ et les statuts d'Eudes de Paris (1208), avant de l'être par le quatrième concile de Latran (1215).

Les sources nationales de l'histoire ecclésiastique médiévale sont loin d'être aussi nombreuses en Suède que dans le reste de l'Europe. Elles se limitent à quelques statuts et sermons synodaux, ainsi que des notices sur des synodes diocésains ${ }^{14}$. Contrairement à la France ou à l'Angleterre, il ne subsiste pratiquement aucun registre ou autre document des cours ecclésiastiques ${ }^{15}$, et les statuts restent l'une des rares sources sur l'application du droit canonique en Suède.

Au Moyen Âge, la Suède constituait une province ecclésiastique divisée en sept circonscriptions: l'archidiocèse d'Upsal et les diocèses de Linköping, Skara, Strängnäs, Västerås, Växjö et Åbo, ce dernier comprenant l'actuelle Finlande ${ }^{16}$. Ces diocèses avaient l'obligation de tenir des synodes annuels comme l'avait prescrit le quatrième concile de Latran ${ }^{17}$. Les synodes diocésains suédois avaient lieu habituellement au même moment que le lagting, la diète provinciale. Il en était de même en Norvège et en Islande, mais pas au Danemark où l'on tenait ces synodes en automne, comme sur le reste du continent. Le concile provincial de Skänninge en 1248 , sous la direction du cardinal Guillaume de Sabine, ainsi que celui de Tälje en 1279, sous la présidence de l'archevêque de Suède Jacobus Israelis, précisent que les évêques étaient tenus à publier des statuts dans

12. Les statuts de 1076 allaient jusqu'à déclarer des mariages sans cérémonie religieuse invalides: «Praeterea statutum est, ut nullus filiam suam, vel cognatam det alicui absque benedictione sacerdotali; si aliter fecerit, non ut legitimum conjugium sed ut fornicatorium

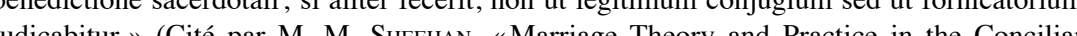
Legislation and Diocesan Statutes of Medieval England», dans Marriage, Family, and Law in Medieval Europe, Toronto, 1996, p. 118-176, ici p. 120.)

13. «Ac illud praeterea constituit ut profani homines publice in templis matrimonio conjungerentur» (Sacrorum conciliorum nova et amplissima collectio, éd.J. D. MANsI, t. 22 , col.674)

14. Les statuts synodaux suédois ont été publiés par H.ReuTERDAHL, dans Statuta synodalia veteris ecclesiae sveogothicae, Lund, 1841, et J.GUMmERus, dans Synodalstatuter och andra kyrkorättsliga aktstycken från den svenska medeltiden, Uppsala, 1902. Un S. Kroon, Statuter från svens sats des conciles provinciaux a récem

15. L'exception étant le registre de Hans Brask, évêque de Linköping, du début du xVI ${ }^{\mathrm{s}}$ siècle: Biskop Hans Brasks registratur, éd.H. GunNENG, Uppsala, 2003.

16. L'organisation diocésaine suédoise s'est développée autour de l'an 1100.Un répertoire florentin des environs de 1120 mentionne les diocèses de Skara, Linköping, Eskilstuna, Strängnäs, Sigtuna et Västerås. Diplomatarium sueconum, Appendix, Acta pontificum Suecia 1: 1, n. 4. Voir aussi S.TUNBERG, «En romersk källa om Norden vid 1100-talets början», Språkvetenskapliga sällskapets i Uppsala förhandlingar 1910-12, Uppsala, 1912.

17. Concilium Laterense [5], éd. MaNsi, t. 22, col. 991. leurs synodes ${ }^{18}$. Ces statuts avaient force de loi dans l'étendue du diocèse et devaient être strictement observés.

Comme beaucoup d'autres institutions de l'Église en Suède, les synodes diocésains furent formés d'après des modèles en vigueur en Europe et l'influence de la France fut particulièrement marquée. La terminologie indique déjà cette influence: comme en France, le synode diocésain est exclusivement nommé synodus; ceci le distingue de termes comme conventio ou capitulum, qui étaient fréquemment utilisés en Allemagne ${ }^{19}$. L'unique ordo synodalis du Moyen Âge suédois qui ait été conservé - dans un registre de la cathédrale d'Upsal de l'année 1344 - présente de grandes ressemblances avec un ordo synodalis de Meaux ${ }^{20}$. L'influence française est encore plus marquée dans l'un des premiers statuts synodaux suédois connus : celui de Brunolphe de Skara.

\section{Les statuts d'Eudes de Paris et de Brunolphe de Skara}

En France, le premier livre synodal connu constituant une véritable législation diocésaine est celui d'Eudes de Sully,évêque de Paris, au tournant du XIII ${ }^{\mathrm{e}}$ siècle. La première partie comprend de courtes règles pour le synode et six chapitres sur les sacrements, sans prendre en compte le sacrement de l'ordre. La deuxième partie, sous la rubrique Incipiunt communia a precepta synodalia, contient des préceptes courants portant, entre autres, sur la discipline du clergé ${ }^{21}$. Les statuts d'Eudes eurent une portée considérable. Ils furent la source principale des statuts synodaux du Nord de la France au XIII ${ }^{\mathrm{e}}$ siècle et marquèrent de nombreux statuts en dehors de la France ${ }^{22}$. Ainsi, par exemple, ils furent utilisés dans les compilations de Trèves et de Mayence, et Richard Poore, évêque de Salisbury, leur emprunta dix-sept chapitres, qui passèrent à leur tour dans d'autres statuts anglais ${ }^{23}$.

18. « hec omnia singulis annis in suis synodis diligenter legi faciant \& exponi»: Concile de Skenninge, Diplomatarium sueconum (ci-dessous $D S$ ), 359. Pour le concile de Tälje, voir DS 692 .

19. S. Kroon, Det svenska prästmötet under medeltiden. Dess uppkomst och ställning $i$ samhälle och kyrka, Lund, 1948, p. 30-31.

20. Ibid., p. 40-41.

21. Pour une description détaillée des statuts d'Eudes de Paris, voir O. Pontal, Les Statuts synodaux français du XIII' siècle, t. 1, Les Statuts de Paris et le synodal de l'Ouest, Paris, 1971, p. 5-50.

22. O. PonTAL, «Les plus anciens statuts synodaux d'Angers et leur expansion dans les diocèses de l'Ouest de la France», Revue d'histoire de l'Église de France, 143 (1960), p. 54.

23. G. Le BRAS, «Paris, seconde capitale de la chrétienté», Revue d'histoire de l'Église de France, 129 (1951). Pour les statuts de Salisbury, voir C. R. CHENEY, English Synodalia of the Thirteenth Century, Londres, 1968, p. 56. 
L'influence des statuts de Paris s'étendit jusqu'en Scandinavie. On en voit la trace dans les statuts de l'archevêque norvégien Eilif Arnesøn, de 1320 , et dans ceux d'Ulrik Stykke, évêque d'Aarhus, de $1443^{24}$. Le type Eudes des statuts diocésains est représenté, en Suède, par les statuts de Strängnäs, une version abrégée de ceux-ci ayant fait partie d'un compendium du Xve siècle, et surtout par les statuts de Skara. Ces derniers, qui datent probablement de la période de Brunolphe $\mathrm{I}^{\mathrm{er}}(1278-1317)$ et sont parmi les plus anciens conservés en Suède, correspondent presque mot pour mot aux statuts d'Eudes de Paris. Brunolphe avait passé pas moins de dixhuit ans à l'Université de Paris avant d'être élu évêque à Skara et il est donc probable qu'il en ait eu connaissance ${ }^{25}$.

À titre de comparaison, nous reproduisons dans le tableau ci-dessous le premier paragraphe sur le mariage de chacun des deux statuts, tels qu'ils ont été retranscrits par Odette Pontal et Jaakko Gummerus respectivement:

\begin{tabular}{|c|c|}
\hline Statuts de Paris & Statuts de Skara \\
\hline $\begin{array}{l}\text { Matrimonium cum honore et } \\
\text { reverentia celebratur [et in facie } \\
\text { Ecclesie], nec cum risu et jocose } \\
\text { ne contempnatur. Et antequam fiat, } \\
\text { semper in tribus dominicis aut tribus } \\
\text { festivis diebus a se distantibus, quasi } \\
\text { tribus edictis, perquirat sacerdos a } \\
\text { populo sub pena excommunicationis } \\
\text { de legitimitate sponsi et sponse qui } \\
\text { debent conjungi et ante fidem datam } \\
\text { de contrahendo matrimonio: et ante } \\
\text { hec tria edicta nullus audeat aliquo } \\
\text { modo matrimonia celebrare. }\end{array}$ & $\begin{array}{l}\text { Matrimonium omni honore et } \\
\text { reuerencia celebretur et in facie } \\
\text { ecclesie, non cum risu et ioco, } \\
\text { ne contempnatur. Antequam fiat, } \\
\text { tribus dominicis diebus vel festiuis } \\
\text { pupplicetur; et perquirat sacerdos } \\
\text { sub pena excommunicacionis de } \\
\text { legittimitate sponsi et sponse, qui } \\
\text { debent coniugi, et ante hoc nullus } \\
\text { audeat matrimonium celebrare. }\end{array}$ \\
\hline $\begin{array}{l}\text { Éd. O. Pontal, Les Statuts synodaux } \\
\text { français du XIII siècle, vol. 1, Les } \\
\text { Statuts de Paris et le synodal de } \\
\text { l'Ouest, Paris, } 1971 \text {, p. 66-68. }\end{array}$ & $\begin{array}{l}\text { Éd. J. Gummerus, Synodalstatuter } \\
\text { och andra kyrkorättsliga aktstycken } \\
\text { från den svenska medeltiden, } \\
\text { Uppsala, } 1902 \text {, p. } 62-63 \text {. }\end{array}$ \\
\hline
\end{tabular}

Comme on peut le constater, le contenu des deux extraits est pratiquement identique; la principale différence étant que le texte de Skara est légèrement abrégé. Les deux statuts prescrivent que le mariage doit

24. Samling af danske Kirke-Love, éd. G. J. ThовкеLIN, Copenhague, 1781, p. 51-84.

25. Den helige biskop Brynolfs av Skara levnad jämte hans kanonisationsprocess, éd. S. BLOMgren, Skara, 1998, p. 16. Pour les années à Paris de Brunolphe, voir B. WAHLSTRÖM, «Studentliv i medeltidens Paris», dans J. HAGBERG, éd., Biskopen och törntaggen, Skara, 2004, p. 219-237. être célébré «avec honneur et respect», et qu'il doit être précédé de trois bans publiés à des jours de fête, afin que le prêtre puisse s'informer de la situation légitime du couple. Les paragraphes qui suivent se ressemblent tout autant: les prêtres doivent interdire aux laïcs de se marier autrement que devant l'Église et en présence du prêtre. Le lieu où le mariage doit être célébré, devant les portes de l'église, est qualifié de «fréquenté» (celebri) dans les statuts de Paris, tandis que ceux de Skara le qualifien d' «approprié» (debito). Dans le texte de Paris, on ajoute la présence de témoins ${ }^{26}$. Les deux statuts interdisent toute espèce de sortilèges pendant les noces, ainsi que de cacher des empêchements. Les statuts de Paris ajoutent la peine d'excommunication pour ceux qui emploient des sortilèges pendant les noces et précisent quels sont les empêchements ${ }^{27}$. En cas de doute sur la légitimité d'un mariage, les deux statuts enjoignent au prêtre de toujours en référer à l'évêque avant de le célébrer ${ }^{28}$. Enfin, les deux statuts interdisent aux prêtres d'exiger quoi que ce soit avant de célébrer le mariage: les statuts de Paris sous peine de suspens, et ceux de Skara sous peine d'excommunication. Les statuts de Paris ajoutent cependant que le prêtre devait recevoir, et pouvait même exiger, des dons une fois le mariage célébré $^{29}$. Dans ce chapitre sur le mariage, le seul paragraphe des statuts de

26. «Prohibeantur frequenter laicis presbyteri, ne dent sibi fidem mutuo de contrahendo, nisi coram sacerdote et in loco celebri, scilicet ante januas ecclesie et coram pluribus» [4 ] (éd. O. PontaL, Les Statuts de Paris..., p. 66). «Prohibeant frequenter laicis clerici ne fidem dent mutuo de contrahendo matrimonio, nisi coram sacerdote et in loco debito, id est, ante ianuas ecclesie» [10] (éd. J. Gummerus, Synodalstatuter..., p. 63).

27. «Sepe in nuptiis prohibeantur per excommunicationem sortilegia fieri; malefici quoque et celantes consanguinitatem et alia impedimenta matrimonii: votum, ordinem, consanguinitatem, affinitatem, disparem cultum, compaternitatem que tantum quatuor a matrimonio excludit personas: compatrem, commatrem, filiolum et fratrem vel sororem spiritualem, scilicet filium vel filiam patrini » [42] (éd. O. PontaL, Les Statuts de Paris...,p. 6668). «In nupciis prohibeantur fieri sortilegia et maleficia, id est celantes consanguinitatem et alia impedimenta matrimonii » [10] (éd. J. GuMmERus, Synodalstatuter..., p. 63).

28. «Nullus sacerdos audeat perficere matrimonium in casu dubio, inconsulto episcopo sed ad eum semper referat omnes matrimonii <dubitationes vel> dubietates » [43] (éd. O. PoNTAL, Les Statuts de Paris . p. 68). «Nullus sacerdos in casu dubio audeat perficere matrimoni inconsulto episcopo, videlicet ad eum semper referat omnes metimoni dubietates " [11] (éd. J. GuMmerus, Synodalstatuter..., p. 63).

29. «Prohibetur districte sub pena suspensionis, ne ullus sacerdos aut capellanus exigat aliquid ante benedictionem nuptialem sive pro testimonio ferendo sive pro matrimonio celebrando, occasione ferculorum que debentur pro nuptiis; celebrato autem matrimonio, recipiat fercula sua et exigat, si necesse fuerit » [44] (éd. O. Pontal, Les Statuts de Paris... p. 68). « Prohibetur eciam districte sub pena excommunicacionis, ut nullus sacerdos aut capellanus exigat aliquid ante benedictionem sponsalem pro matrimonio celebrando» [12] (éd. J. Gummerus, Synodalstatuter..., p. 63). 
Paris qu'on ne retrouve pas dans ceux de Skara concerne l'interdiction faite aux époux d'entrer en religion ${ }^{30}$.

Cependant, les autres chapitres de ces statuts introduisent une différence essentielle pour le mariage. Dans la quatrième partie des statuts de Paris, on trouve deux paragraphes sur les mariages clandestins: le premier interdit aux prêtres de les célébrer ou de les bénir sous peine d'excommunication, et le deuxième frappe d'une même excommunication ceux qui se marient ou font bénir leur mariage clandestinement, comme ceux qui y assistent ${ }^{31}$. Le passage des statuts de Skara qui y correspond le mieux affirme que ceux qui refusent de faire bénir leur mariage doivent être suspendus de l'eucharistie: «Si des mariés ne veulent pas bénir leur mariage, ils seront suspendus de l'eucharistie, et si l'un veut et l'autre pas, celui qui ne veut pas sera suspendu ${ }^{32}$.»Il ne s'agit donc pas, comme dans les statuts de Paris, de mariage clandestin proprement dit, mais plutôt du refus de célébrer religieusement un mariage déjà contracté selon le droit séculier.

\section{Mariages clandestins}

La lutte contre les mariages clandestins était une des préoccupations majeures des synodes diocésains français. Le concile de Latran avait prescrit des règles générales quant au caractère public du mariage, mais c'est dans les statuts synodaux que les modalités exactes pour y parvenir se précisent. Pratiquement tous les statuts français $\mathrm{du} \mathrm{XIII}^{\mathrm{e}}$ siècle répètent l'obligation d'annoncer le mariage publiquement, avec quelques variations quant aux peines encourues en cas de refus. Les statuts prévoient la suspension, pouvant aller jusqu'à trois ans et souvent associée à d'autres peines, pour les prêtres qui célébreraient des mariages clandestins. Quant aux contractants, ils encouraient l'excommunication, des amendes ou d'autres peines à la discrétion du juge. Selon de nombreux statuts, le simple fait d'assister à des mariages clandestins faisait courir le risque d'excommunication ${ }^{33}$. Malgré

30. «prohibeant sacerdotes in ecclesia publice sub excommunicatione ne alter conjugum transeat ad religionem, aut recipiatur, nisi per episcopum vel archidiaconum» [45] (éd. O. PontaL, Les Statuts de Paris..., p. 68).

31. "Item districte precipitur presbyteris et sub excommunicatione, ne aliquas matrimonio clandestino conjungant vel benedicant» [97] ; «Item excommunicentur omnes matrimonio clandestino conjungant vel benedicant » $[97]$; «Item excommunicentur omnes [98] (éd. O. Pontal, Les Statuts de Paris..., p. 88).

32] (éd. O. PONTAL, Les Statuts de Paris..., p. 88).
32 coniuges noluerint benedici contracto matrimonio, suspendantur a corpore Christi, et si alter voluerit et alter negauerit, negans suspendatur » [30] (éd. J. GumMERus, Synodalstatuter..., p. 65).

33. Voir par exemple les statuts de Nicolas Gellent [8], éd. J. AvRIL, Les Statuts synodaux..., t. 3, Les Statuts synodaux angevins de la seconde moitié du XIII siècle, Paris, 1988, p. 78, et les statuts de Guillaume le Maire [1], ibid., p. 214-216. toutes ces dispositions, les laïcs continuaient à se marier clandestinement Le synode de Coutances (1300) note avec un certain désabusement qu'aussi bien les prêtres que les laïcs se montraient souvent négligents au regard de ces interdictions. Ils les transgressaient sans craindre apparemment les peines prévues ${ }^{34}$. En butte à ces échecs, les évêques persévèrent et, dans un synode de la même ville en 1372, ils réitèrent la peine de suspension pour les prêtres et d'excommunication pour les laïcs assistant à des mariages clandestins ${ }^{35}$.

La publication de bans et le caractère public du mariage étaient essentiels pour connaître à l'avance d'éventuels empêchements. Ils l'étaient aussi pour établir clairement le statut matrimonial des laïcs afin d'éviter de longs litiges et d'éventuelles unions bigames. Comme Richard Helmholz, entre autres, l'a fait remarquer, les causes matrimoniales pour prouver l'existence d'un mariage étaient bien plus fréquentes que celles pour dissoudre ou invalider un lien ${ }^{36}$.

En comparaison avec la France, et d'ailleurs avec la plupart des autres pays européens, il y a relativement peu de références aux mariages clandestins dans les statuts synodaux suédois. De plus, ces dernières visent surtout l'absence de cérémonie religieuse, et non son caractère public. La plupart des statuts prescrivent la publication de bans et la bénédiction nuptiale. Les mariages clandestins dans le sens de mariages canoniquement valides, contractés par consentement mutuel, sans témoins, ni cérémonie publique, ne sont mentionnés que dans quelques passages: le synode provincial de Tälje (1368) interdit aux prêtres de bénir des fiançailles clandestines (clandestinis desponsationibus), et les statuts de Linköping interdisent aux mêmes prêtres d'assister aux mariages clandestins ${ }^{37}$. Le compendium Upsaliensis provincie statutorum de Nicolaus Ragvaldi

34. «quia tamen in hujusmodi canonis observatione quamplurimos vidimus sæpius negligentes, immo verius transgressores, penas non timentes prædictas, viasque exquirente \& modos per quos ostenta sibi impedimenta devitent» (Concilia Rotomagensis provincia : accedunt Dioecesance synodi, pontificum epistola, regia pro normannice clero diplomata, éd. G. BEssin, Rouen, 1717, p. 559).

35. «Statuimus insuper ne nulla persona ecclesiatica vel sæcularis, cujuscumque statu vel conditionis existat, matrimonium clandestinum in nostri civitate \& Diocesi, bannis nondum factis per tres Dominicos, ut moris est, nisi de nostra licentia celebrare præsumat. $\mathrm{Si}$ quis vero contra fecerit, aut operam, concilium vel favorem prestiterit, suspension Si gis vero con fece sententiam inctit . principas apen consilii \& favorem in

bus prastid., p. 562).

36. R. H. HeLMHOLZ, Marriage Litigation in Medieval England, Cambridge, 1975, p. 75 37. «Item nullus sacerdos præsumat interesse clandestinis desponsationibus seu ante ternam proclamationem in eccl: a benedicere taliter desponsatos sub pena suspensionis officii et beneficii » (éd. H. Reuterdahl, Statuta synodalia..., p. 56). « Item non celebrent clandestina matrimonia, nec eis intersint, poena excommunicationis » (Statuta Lincopensia [25], ibid., p. 77). Les statuts de Linköping notent aussi, parmi les causes qui devraient toujours être remises au jugement de l'évêque, le cas où quelqu'un déjà marié par verba de 
(1441), une compilation des statuts des conciles provinciaux d'Upsal ${ }^{38}$, ne traite pas du mariage clandestin. On y prescrit le jour où l'on peut bénir un mariage, qu'on ne doit pas donner cette bénédiction si la mariée est veuve, qu'un couple fiancé n'a pas le droit de dormir ensemble avant la bénédiction nuptiale (sous peine d'une amende de trois marcs) et que les étrangers doivent pouvoir prouver qu'ils ne sont pas déjà mariés ${ }^{39}$. Ces deux dernières règles visent d'une certaine manière les mariages clandestins: la première évite un mariage présomptif et la deuxième concerne le risque de bigamie si le statut marital des contractants n'est pas clairement établi. Cependant, il n'y a pas de référence directe aux mariages clandestins, comme par exemple l'interdiction de contracter, célébrer ou assister à de tels mariages, qui sont plutôt de règle dans les statuts synodaux français. Il est aussi notable qu'aucune loi séculière suédoise de cette époque ne mentionne les mariages clandestins.

Même lors de références directes aux mariages clandestins, ce qui est réellement visé n'est pas toujours clair. La Summa canonum variorum conciliorum définit comme clandestins les mariages qui se font «sans témoins, sans solennité et sans publication de bans précédents ${ }^{40} »$. Dans nos statuts, c'est presque exclusivement les deux dernières obligations qui sont explicitement requises, la présence de témoins semblant aller de soi. En pratique, l'expression «mariage clandestin» signifiait en Suède que des témoins des deux familles étaient présents à une fête publique, mais qu'on avait omis la publication de bans et la bénédiction nuptiale ${ }^{41}$.

Si les mariages clandestins semblent relativement peu présents dans les statuts synodaux suédois, la situation maritale des étrangers et inconnus (advene et ignoti) occupe en revanche une place importante. Ce problème,

presenti, se mariait publiquement avec une autre : ibid.,p. 82. Voir aussi les ordonnances de visitation épiscopale de la même ville en 1480 : J. GuMMERus, Synodalstatuter..., p. 97.

38. Ce texte est né du désir d'harmoniser les nombreux statuts provinciaux qui s'accumulent depuis le concile provincial de Skenninge en 1248. Ils sont obscurs et parfois contradictoires. En 1441, l'archevêque Nicolaus Ragvaldi demande à l'évêque de Strängnäs de les unifier en un compendium : «Upsaliensis provinciae statuta eadem in unum compendium redigere » (cité dans S. Kroon, Statuter..., p. 7).

39. « Nupciarum benedictio fiat tantum in ultimo festo, ubi plura concurrunt, et contrafaciens in tribus marcis puniatur. Quando vir alias benedictus contrahit cum virgine vel corrupta alias non benedicta, nupcie solenniter benedicantur. Sed si vidua contrahit cum venedicto sive non benedicto, no benedicantur (Compendium (Upsaliesis prouiciae 450), netli desposat adialium psaliensis proninciae, 4.50). « Nulli desponsati adinicen donniant ante benedictionem sub pena iii marcarum » (ibid., 4:36). "Advene non admittantur ad contrahendum matrimonium, nisi per literas docuerint se solutos. Et sacerdos, qui tales benedicit, in iii marcis puniatur » (ibid., 4:35, S. Kroon, Statuter..., p. 88, 80 et 79)

40. « clandestinum autem dicitur, cum non habentur testes, nec fiunt cum solennitate, nec praemissis bannis » (Summa canonum variorum conciliorum,éd. H. ReUTERDAHL, Statuta synodalia..., p. 192).

41. M. Korpiola, Between Betrothal and Bedding..., p. 147. inexistant dans les statuts de Paris et ceux de Skara, prend une place considérable dans les statuts suédois plus tardifs, qui prévoient différentes peines pour ceux qui seraient incapables de prouver leur statut marital. Selon les statuts du Jämtland de l'archevêque Jöns Håkansson (1425) ceux qui venaient en couple et se disaient mariés n'avaient pas le droit de recevoir l'eucharistie à Pâques s'ils ne pouvaient pas prouver, par une lettre ou un document équivalent, qu'ils étaient réellement mariés. Pour avoir le droit de se marier sur place, un étranger devait pouvoir prouver qu'il n'était pas déjà marié ${ }^{42}$. Le prêtre qui bénissait malgré tout un tel mariage devait, selon les statuts d'Arboga de Johannes Jerechini, payer une amende de trois marcs ${ }^{43}$. Selon d'autres statuts de la même ville (1417), les étrangers qui amenaient des femmes sans pouvoir démontrer qu'ils étaient mariés n'avaient ni le droit d'assister à la messe, ni de recevoir l'eucharistie, sau au seuil de la mort ${ }^{44}$. Les mêmes peines et l'interdiction d'assister à la messe se retrouvent dans le Summa canonum variorum conciliorum et dans le compendium Upsaliensis provincie statutorum ${ }^{45}$.

Dans les statuts français, les références à la situation maritale des étrangers et inconnus sont plus rares. Il en existe quelques cas : les statuts de Pierre de Sampson, par exemple, interdisent qu'un prêtre marie des personnes qui lui sont inconnues et les statuts de Gellent soulèvent le problème de façon indirecte en interdisant aux prêtres d'exiger des gratifications pour écrire des lettres prouvant qu'un couple est marié ${ }^{46}$. Le problème est cependant bien moins présent que dans les statuts suédois. Une explication possible est que ce type de situation était couvert, pour une large part, par les statuts visant les mariages clandestins en général que l’on trouve dans la

42. «item viliom wj at farande folk. som aff androm landom komber oc sic for hionalagh halla ey sculu redhskap om pascha faa. for æn the breff oc skæl haua. at the laglica saman komin æra. fore thy margher kombir löpande mz frænko sinne eller annars hustro Oc williom komin æra. fore thy margher kombir löpande $\mathrm{mz}$ frænko sinne eller annars hustro Oc williom
wi ath ey noghor wtlændsker til giffto stædhias scal wtan han full skæl oc bewiisning hafuir wi ath ey noghor wtlændsker til giffto stædhias scal wtan han full skæl oc bewiisning hafuir
thz han ey annarstads giffther ær. Oc thetta biudhom wi sunnerligha allom warom klerchom thz han ey annarstads giffther ær. Oc thetta biudhom wi sunnerligha allom warom klerchom
granelica war at tagha » (éd. J. Gummerus, Synodalstatuter..., p. 44).

43. "Item quod advene et ignoti non admittantur ad contrahendum matrimonia nisi super statu suo quod sint soluti per literas aut testes docuerint evidenter, sacerdos autem qui tales benedixierit puniatur in tribus marchis » (Statutum Johannes Jerechini Arbogae [4], éd. H. ReUTERDAHL, Statuta synodalia..., p. 107).

44. «Item statuimus quod layci advene et ignoti adducentes secum aliquas mulieres ad parochias curatorum nisi literis vel testibus docuerint se esse legitime coniunctos non permittantur divinis interesse, nec ad eucharistie perceptionem nec* in mortis articulo $\left(*^{*}\right.$ nec $»$ doit très probablement être remplacé par « nisi ») (Statuta concilii arbogensis 1417). Cf. Compendium..., 4:35, S. Kroon, Statuter..., p. 79 ; voir aussi H. Reuterdaht, Statuta synodalia..., p. 155 et 158

45. H. ReUTERDAHL, Statuta synodalia ..., p. 182 ; S. KROON, Statuter ..., p. 79

46. Synodal de Nîmes, Arles, Béziers, etc., éd. O. PonTaL, Les Statuts synodaux..., t. 2 , Les Statuts de 1230 à 1260, Paris, 1983, p. 390. Statuts de Gellent, éd. J. AvRIL, t. 3, p. 98 100 . 
plupart des statuts synodaux français. Les statuts concernant les mariages clandestins étaient plus rares en Suède et les évêques se sentaient dans la étrangers et inconnus.

\section{Les statuts synodaux et le droit séculier}

En Suède, le droit matrimonial était réglé non seulement par le droit ecclésiastique, mais aussi par le droit séculier. Les lois provinciales édictaient des règles détaillées pour les fiançailles et les mariages contenues dans le giftasbalk, «livre de mariage», ou dans le ärvdabalk, «livre de successions», ou encore dans le kyrkobalk, «livre de l'Église».Le kyrkobalk couvrait tout ce qui concernait l'Eglise et se fondait sur les statuts synodaux plus ou moins accommodés aux intérêts séculiers. Les statuts synodaux proprement dits, le latinbalk, souvent rédigés en latin (d'où le nom de «livre en latin»), constituaient la base de la juridiction ecclésiastique. Les et deux cours de justice: soit, suivant le kyrkobalk, par la diète, soit, suivant le latinbalk, par l'évêque.

Le mariage, dans le droit séculier suédois, était un processus lent par lequel les époux passaient graduellement de l'état de non-mariés à l'état de mariés. Les étapes principales étaient les fiançailles, le mariage, les noces, le coucher ${ }^{47}$ et le morghongäf, cadeaux faits par le mari à sa femme le matin suivant les noces ${ }^{48}$. À ces règles s'ajoutaient celles du droit de l'Église, portant principalement sur la publication de bans et la bénédiction nuptiale.

Le tableau ci-dessous résume les règles principales de formation du mariage telles qu'elles ont été respectivement transcrites dans les livres nécessité d'enquêter plus systématiquement sur la situation maritale des questions concernant l'Église pouvaient être jugées en réalité par deux lois

de succession ou de mariage, les livres de l'Église ${ }^{49}$ et dans les statuts synodaux :

\begin{tabular}{|c|c|c|}
\hline $\begin{array}{l}\text { Livre d } \\
\text { ou de } n\end{array}$ & Livre de l'Église & Statuts synodaux \\
\hline $\begin{array}{l}\text { L'homme doit aller } \\
\text { trouver le père et les } \\
\text { plus proches parents } \\
\text { de la femme et requérir } \\
\text { leur consentement. Ầ ce } \\
\text { moment, le giftoman est } \\
\text { désigné. } \\
\text { Le giftoman doit fiancer } \\
\text { la femme avec un nombre } \\
\text { prétabli de témoins des } \\
\text { deux côtés } \\
\text { Le } \\
\text { Le giftoman donne la } \\
\text { femme à son mari avec } \\
\text { la formule suivante : je } \\
\text { te la donne pour épouse } \\
\text { honorable, pour partager } \\
\text { ton lit, pour les serrures } \\
\text { et les clefs et avec le } \\
\text { tiers légal dans tous les } \\
\text { meubles que tu possèdes } \\
\text { et que tu peux acquérir }{ }^{51} \text {. } \\
\text { La mariée est amenée au } \\
\text { lit de son mari. C'est àce } \\
\text { moment qu'il devient son } \\
\text { tuteur légal. } \\
\text { Le lendemain des noces } \\
\text { au matin, le mari donne à } \\
\text { sa femme la morghongaff } \\
\text { en présence de témoins. }\end{array}$ & $\begin{array}{l}\text { Pour le mariage ainsi que } \\
\text { l'inceste, l'évêque seul } \\
\text { peut juger. } \\
\text { Si des fiançailles sont } \\
\text { rompues par un jugement } \\
\text { ecclésiastique, l'évêque } \\
\text { perçoit une amende } \\
\text { pour chaque séparation, } \\
\text { payable par le responsable } \\
\text { de la séparation. } \\
\text { Si un homme et une } \\
\text { femme veulent se marier à } \\
\text { l'église, ils doivent le faire } \\
\text { savoir au prêtre de leur } \\
\text { paroisse. Le prêtre doit } \\
\text { publier trois dimanches } \\
\text { à la porte de l'église que } \\
\text { le mari et la femme sont } \\
\text { fiancés conformément à la } \\
\text { loi de la province et qu'ils } \\
\text { veulent faire bénir leur } \\
\text { union conformément au } \\
\text { droit de l'Église. } \\
\text { Si quelqu'un connaissant } \\
\text { un empêchement vient } \\
\text { avant le jour fixé, le prêtre } \\
\text { ne peut pas les bénir avant } \\
\text { que l'affaire n'ait été } \\
\text { élucidée. }\end{array}$ & $\begin{array}{l}\text { Le mariage doit être } \\
\text { célébré avec honneur et } \\
\text { respect devant l'église. } \\
\text { Il doit être publié trois } \\
\text { dimanches ou trois jours } \\
\text { de fête ; et le prêtre doit } \\
\text { s'informer de la situation } \\
\text { légitime du fiancé et de la } \\
\text { fiancée. } \\
\text { Les clercs doivent } \\
\text { interdire aux laïcs de se } \\
\text { donner le consentement } \\
\text { mutuel de contracter } \\
\text { mariage, si ce n'est devant } \\
\text { un prêtre et devant les } \\
\text { portes de l'église. } \\
\text { Il est interdit de faire } \\
\text { des sortilèges dans } \\
\text { les noces et de cacher } \\
\text { des empêchements au } \\
\text { mariage. } \\
\text { Il est interdit qu'un } \\
\text { prêtre ou chapelain exige } \\
\text { quoi que ce soit avant la } \\
\text { bénédiction nuptiale pour } \\
\text { célébrer le mariage. }\end{array}$ \\
\hline
\end{tabular}

49. Le texte sur le mariage dans le droit séculier est principalement tiré de la loi d'Upland, 47. Le coucher de la mariée dans le lit de son mari signifiait qu'il devenait son tuteur légal : «Komber brup i siæng mæp bondæ sinom. pa aa han foræ hana bapæ swaræ oc sökiæ » et la loi de Vestmanie ( $\ddot{B}$ B 2:1). La loi d'Upland le décrit comme le moment où la femme rentre chez son mari : «nu kombær bruð hem til bondæ sins » ( e il est probable qu'il s'agit en fait du coucher. Voir L. CARLSSON, Jag giver dig min dotter... vol. 1, p. 143-44.

48. Elle correspond à la Morgengabe germanique. Les diplômes la nomment donacio propter nuptias et elle a pour but d'honorer la femme : « ex parte domini Nycholai quondam Mychiæls sun. Pro donacione propter nupcias wlgariter Morhons Giæf sibi assignata per ipsum N. tunc maritum suum »(DS 811). Voir aussi $D S 541$ et 588. La donation avait lieu solennellement en présence de témoins et devenait la propriété privée de la femme. « Noverint vniuersi me dilecte consorti mee ingridi pro honore et racione dotis quod wlgariter dicitur hinderdagsgif bona mea in sioarydh et hardanes dedisse » (DS 3364). Voir aussi DS 3954 qui est la loi provinciale suédoise la plus détaillée, et aussi celle qui a le plus influencé les lois provinciales plus tardives, ainsi que la loi nationale de Magnus Eriksson.

50. Le nombre de témoins pouvait varier selon les différentes lois : la loi d'Upland et la loi de Helsinge en prescrivent quatre de chaque côté (ÄB $1: 1$ ); la loi de Dalécarlie, deux (GB et ÄB 1) ; et la loi de la ville de Magnus Eriksson, six (GB 2).

51. Loi d'Upland (ÄB 3). La formule, reprise dans la loi de Vestmanie (ÄB 3), indique clairement la position de la femme comme épouse honorable avec la responsabilité du foyer (clefs et serrures). Le droit du tiers légal établit le droit de la femme à un tiers de tous les meubles. La loi d'Upland fait l'exception de l'or et des esclaves qui restaient la propriété exclusive du mari. Voir C. ChRISTENSEN-Nugues, « Äktenskap och familj », p. 301. 
Le fait le plus marquant dans le droit séculier est le nombre de règles, rituels et cérémonies qui entourent le mariage. Chaque étape de la formation du mariage est soigneusement décrite, les témoins nécessaires désignés et les formules à utiliser précisées. Que le mariage soit entouré de rites et de cérémonies n'est pas particulier à la Suède ; c'était le cas dans la plupart des pays européens. Ce qui est remarquable, c'est que ces rites et cérémonies furent prescrits par la loi.

Dans le livre de l'Église, le droit de l'Église est en quelque sorte subordonné au droit séculier ${ }^{52}$. On note au départ que seul l'évêque peut juger des cas de mariages et d'incestes, mais ce qui suit semble plutôt diminuer le rôle de l'Église. La bénédiction nuptiale est facultative et ne peut pas se faire avant que le couple soit fiancé selon la loi de la province. La bénédiction nuptiale n'est prescrite que par la loi d'Ostrogothie (GB 6). Les fiançailles étaient une étape très importante dans la formation du mariage selon le droit séculier suédois, et ne doivent pas être confondues avec le simple desponsatio du droit canonique. C'était au moment des fiançailles que l'accord entre les deux familles était scellé et que l'on décidait du montant de la dot. Par ailleurs, les fiançailles se faisaient toujours en présence de témoins. La nécessité de se fiancer conformément à la loi de la province avant de faire bénir le mariage à l'église assurait donc qu'il y ait des témoins des deux familles et rendait en principe impossibles les mariages clandestins (dans le sens de mariages secrets).

Le giftoman (littéralement «celui qui donne») avait un rôle très important. Il était le porte-parole de la femme et c'est lui qui avait la responsabilité de son mariage ${ }^{53}$. Le giftoman était désigné par la famille de la mariée, mais selon des critères établis dans les lois régionales. La loi de Dalécarlie (GB) donne les règles suivantes : «Le père est giftoman s'il est en vie. À défaut du père, c'est le frère. S'il n'y a ni père, ni frère, c'est la mère, si elle ne s'est pas remariée. Si elle s'est remariée avec le consentement de ses enfants et des parents de son mari défunt, elle sera giftoman ${ }^{54}$.» Celui

52. Cette subordination du droit de l'Église au droit séculier dans les lois provinciales, qui apparaît aussi dans d'autres domaines, a soulevé des protestations de la part de l'Église : en 1347, cinq clercs contestaient le projet d'inclure un kyrkobalk dans la loi nationale qui était en préparation par le roi Magnus Eriksson. Les clercs craignaient que le pouvoir séculier, en incluant le droit de l'Église dans cette nouvelle loi, l'aurait dénaturé et accommodé à

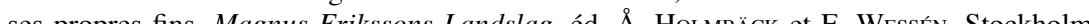

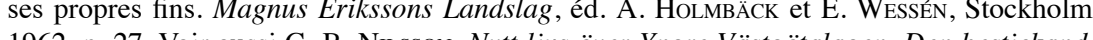
, Jentickande teorin om en medeltida lagstiftningsprocess, Stockholm, 2012, pour la lutte de pouvoir entre l'Église et les intérêts séculiers.

53. Pour le rôle central du giftoman, voir M. KoRPIOLA, Between Betrothal and Bedding..., p. 5-14.

54. Svenska landskapslagar: Dalalagen och Västmannalagen, vol. 2, éd. E. Wessén et Å. HоLмвӓск, Uppsala 1936, p. 78. Le giftoman pouvait donc, malgré la dénomination, aussi être une femme. Selon la loi d'Upland, la loi de Helsinge et la loi de Vestmanie, la mère avait qui portait atteinte aux prérogatives du giftoman s'exposait à de lourdes peines: selon la loi d'Upland, celui qui donnait une femme en mariage sans la permission du giftoman devait payer une amende de quarante marcs ou prouver par le serment de dix-huit co-jureurs qu'il avait l'autorisation de ce dernier ${ }^{55}$. L'interdiction de marier une femme sans l'autorisation du giftoman s'appliquait aussi aux prêtres: «Si un prêtre marie une femme en l'absence du giftoman, il lui vole son droit de marier. Il doit alors payer une amende de quarante marcs, ou prouver par le serment de sept prêtres qu'il avait l'autorisation du giftoman ${ }^{56}$.» La nécessité d'obtenir l'autorisation du giftoman empêchait qu'un couple ne se marie clandestinement pour échapper au contrôle familial - une situation qui n'était pas rare dans le reste de l'Europe ${ }^{57}$.

Les sources nous décrivent le mariage dans la Suède médiévale comme un cérémoniel séculier comportant plusieurs étapes bien établies : fiançailles, don de la mariée, transfert de la mariée à la maison de l'époux, coucher et, enfin, don du matin. Ce mariage par étapes, au rituel scrupuleusement codifié, constituait quasiment l'antithèse du mariage canonique, où seul le consentement des futurs époux, sans autres formalités, était suffisant pour conduire à un mariage valide. Comme Mia Korpiola l'a souligné, l'introduction de la notion de mariage privé, sans cérémonie, contracté seulement par des mots, était étrangère à la culture légale, rituelle et formaliste de la Suède médiévale ${ }^{58}$.

Les traditions, réunies dans les lois séculières, assuraient un caractère public au mariage et la question d'établir s'il avait été contracté ou non, un cas si fréquent sur le continent, n'existait guère en dehors du cas d'étrangers

même précédence sur le frère et prenait ce rôle à défaut du père. Les lois goths, en revanche, ne reconnaissent pas ce droit à la mère ( $c f . \mathrm{Wg}$. II, GB. 2) et, dans la loi nationale de Magnus Eriksson, il est expressément dit que le giftoman ne peut être une femme : «homme et pas femme, mais avec le conseil de la mère » (GB 1$)$.

55. «hwar sum giptir mø utæn rætz giptæ manz orloff. bøte. xl. markr. ællr witi loff aff rættum giptæ manni mz atertan mannæ epi» (Loi d'Upland, ÄB 1. 3). L'office de giftoman pouvait cependant rentrer en conflit avec les intérêts de la personne tenant ce rôle. Comme il était le plus souvent le principal héritier de la femme, il pouvait préférer qu'elle reste célibataire. Ainsi, la loi d'Islande donnait l'autorisation à la femme de se marier san le consentement du giftoman si celui-ci avait refusé deux prétendants convenables. Voir L C

56. «Uighir præstær ok ær egh gipta maprin uipær, pa rænir han gipta mala, pæt ær fiuratighi marka sak ælla siu præsta epær, at han uighpi mæp uilia hins, sum gipta mannin uar » (Loi d'Ostrogothie, GB. 6).

57. Un exemple particulièrement bien documenté est le mariage clandestin entre Margery Paston et Richard Calle, où la famille de Margery a fait tout en son pouvoir pour l'invalider sans y parvenir. Voir Paston Letters and Papers of the Fifteenth Century, éd. N. DavIs, Oxford, 1971

58. M. Korpiola, Between Betrothal and Bedding..., p. 100 
ou d'inconnus. Quand les statuts suédois insistent sur la publication de bans et la bénédiction nuptiale, c'est, d'une part, pour découvrir d'éventuels empêchements, et, d'autre part, pour garantir un plus grand contrôle de l'Église. D'une manière fondamentale, ce n'était pas, comme souvent dans le reste de l'Europe, pour éviter d'éventuels mariages secrets. Par les règles du droit séculier, le mariage en Suède était une affaire publique.

À la fin du Moyen Âge, et surtout à partir du XVI ${ }^{\mathrm{e}}$ siècle, la législation matrimoniale de l'Église catholique est de plus en plus remise en cause. La Réforme protestante la condamne pour avoir fait du mariage «un état faible, méprisé et rejeté ${ }^{59} »$. Selon les Réformés, une des conséquences les plus déplorables de cette législation était que des jeunes gens pouvaient se marier secrètement et contre la volonté de leurs parents. Cette critique n'était nullement confinée à des mouvements protestants. Le statut du mariage était devenu un souci général dans l'Europe renaissante. Un lien quasi organique semblait lier l'institution matrimoniale à la stabilité sociale - l'ordre social reposant sur l'ordre familial ${ }^{60}$. Le manque de règles strictes, ainsi que de contrôle parental et des institutions publiques, semblait menacer cet ordre. La réforme du mariage était devenue une préoccupation aussi bien pour les Catholiques que pour les Protestants ${ }^{61}$

Dans ce contexte, la situation qui avait prévalu dans la Suède médiévale apparaît comme une exception. Bien sûr, la doctrine de consensus s'appliquait en Suède aussi; au moins théoriquement, les principes fondamentaux du droit canonique devaient être respectés dans toute la Chrétienté. Il est aussi évident dans les sources littéraires du XIII ${ }^{\mathrm{e}}$ et XIV ${ }^{\mathrm{e}}$ siècle que cette doctrine fut perçue comme une menace, notamment à cause de la possibilité qui était ainsi donnée aux enfants de se soustraire au pouvoir parental ${ }^{62}$. Cependant, à en juger par les statuts synodaux, cette menace, sur le plan pratique et concret, s'est révélée moins présente qu'ailleurs. Cet

59. «Dieweyl in der Welt der Ehelichen Stand so ubel zerissen/verschmehet und vervorffen wirdt/als ein elender verachter Stand» (Leonard Culman, Jungen Gesellen, Jungkfrauwen und Witwen, Magdebourg, 1534, cité dans S. OzMENT, When Fathers Ruled: Family Life in ReformationEurope, Cambridge (MA), 1983, p. 4).

60. S. Melchior-BonNET, «De la réforme au siècle des lumières. Le monopole de l'Église contesté», dans S. Melchior-Bonnet et C. Salles éd., Histoire du mariage..., p. 433.

61. Pour le lien entre contrôle parental (et public) du mariage et la formation de l'état dans la France du Xvl ${ }^{\mathrm{e}}$ siècle, voir S. HANLEY, «The Jurisprudence of the Arrêts: Marital Union, Civil Society, and State Formation in France, 1550-1650», Law and History Review, 21/1 (2003), p. 1-40.

62. Dans le Gesta danorum de Saxo Grammaticus, les quelques mariages qui sont contractés suivant la volonté de la mariée se terminent tous en catastrophe (SAXo Grammaticus, Gesta Danorum X : 9, éd. J. OLRIK et H. RAEDER, Hauniae, 1932). La même méfiance vis-à-vis de la doctrine du consensus, et notamment la possibilité pour les femmes de choisir ellesmêmes leurs époux, apparaît, entre autres, dans le Heimskringla et le Sverresaga. Voir aussi J. M. Jochens, « Consent in Marriage : Old Norse Law, Life, and Literature », Scandinavian Studies, 58 (1986), p. 142-176. état de fait est sans doute en partie dû à la christianisation tardive de la Scandinavie. Les traditions anciennes, codifiées dans les lois régionales, ont constitué une sorte de législation parallèle à celle de l'Église. Cette législation a donné au mariage son caractère public ainsi qu'une influence décisive de la famille sur les choix matrimoniaux des enfants, notamment par le rôle du giftoman. Paradoxalement, ce «retard» suédois a préfiguré le développement d'un mouvement majeur en Europe au Xvi siècle, c'est-àdire l'entrée du mariage dans la sphère civile et le contrôle plus étroit, à la fois public et parental, de l'institution matrimoniale.

\section{Charlotte CHRISTENSEN-NUGUES - Lund University}

Mariages clandestins dans la Suède médiévale. Le témoignage des statuts synodaux

Selon le droit canonique médiéval, le consentement mutuel était le seul acte nécessaire pour créer un mariage valide; un mariage contracté secrètement pouvait donc lui aussi être parfaitement valide. De tels mariages, dits clandestins, présentaient néanmoins des difficultés pratiques majeures lors de l'application de la législation matrimoniale de l'Église. Ceci était particulièrement apparent au niveau diocésain et presque tous les statuts synodaux européens traitent de ces mariages. Dans ce contexte, les statuts synodaux suédois se distinguent par la place relativement modeste accordée à ce problème.

Nous comparons dans cet article les références aux mariages clandestins dans les statuts synodaux suédois et français et nous explorons les raisons possibles de leurs différences dans la tradition légale suédoise La législation séculière de la Suède médiévale comportait des règles détaillées pour la formation du mariage. Elles avaient comme conséquence de lui assurer une certaine publicité. Ceci faisait que les mariages clandestins, dans le sens de mariages secrets et sans témoins, étaient relativement rares, dans le sen synodaux suédois reflètent cette situation et mettent en rares. Les statuts synodaux suedois refletent cette situation et mettent en lumiere l'influence de traditions matrimoniales antérieures et la manière dont le droit commun
s'en est accommodé. droit canonique - droit séculier - mariage clandestin - statuts synodaux Suède

\section{Clandestine Marriages in the Synodal Statutes of Medieval Sweden}

According to medieval canon law, mutual consent was sufficient to create a valid marriage. A marriage contracted secretly could thus be perfectly valid. Such marriages, i.e. clandestine marriages, did however entail major practical difficulties when the Church's marriage legislation was applied. This was particularly apparent at the local level, and practically all synodal statutes in medieval Europe contained multiple prohibitions against such marriages. In this context, Swedish synodal statutes constitute such marriages. In this context, Swedish synodal statutes constitute This article compares the references to clandestine marriages in Swedish and French synodal statutes, analyses their differences and explores the and French synodal statutes, analyses their differences and explores the Swedish legal tradition to deternine possible explanations for them. that guaranteed a certain publicity. This made clandestine marriages, in the 
sense of marriages contracted secretly without witnesses, relatively scarce. Swedish synodal statutes reflect this situation, shows the implications of the Swedish matrimonial tradition, and how canon law was adapted to the Swedish context.

canon law - clandestine marriage - secular law - synodal statutes - Sweden 